\title{
Optimization of laser-driven intramolecular hydrogen transfer in the presence of dephasing
}

\author{
Eitan Geva \\ Department of Chemistry and The FOCUS Center, University of Michigan, \\ Ann Arbor, Michigan 48109-1055
}

(Received 20 August 2001; accepted 6 November 2001)

\begin{abstract}
The coherent control of laser-driven intramolecular hydrogen transfer is considered in the presence of pure dephasing. Emphasis is put on performing the optimization in the presence of dephasing. Simple analytical expressions are obtained for the optimal pulse shape and optimal yield as functions of the dephasing rate constant. It is found that dephasing is not always uncontrollable and destructive, and that the optimal pulse shape is dictated by the minimization of the destructive attributes of the dephasing processes, as much as by steering the coherent component of the dynamics towards the desired goal. (C) 2002 American Institute of Physics.
\end{abstract}

[DOI: $10.1063 / 1.1431276]$

\section{INTRODUCTION}

The goal of quantum coherent control is the design of laser pulses for controlling molecular motion and chemical reactivity. ${ }^{1-4}$ The main question is formulated in terms of optimal control theory: Given the constraints on the duration and intensity of the pulse, what temporal changes of the amplitude and phase of the laser field would maximize a desired objective? The objective is usually represented by an expectation value at the final state. The optimal pulse is found by solving the optimal control problem, either computationally, via quantum molecular dynamics simulations, ${ }^{5-7}$ or experimentally, via a closed adaptive learning loop. ${ }^{8-10}$

Most of the research to date in coherent control has focused on isolated systems which are not subject to decoherence (decoherence is defined as any nonunitary contribution to the dynamics). Some of the main reasons for this are as follows:

(1) Decoherence is usually conceived as uncontrollable and purely destructive due to its ability to dephase the very same coherences that make coherent control possible in the first place.

(2) Decoherence is assumed to be too slow to be effective on the ultrafast time scale on which the laser pulse operates.

(3) Gas-phase coherent control is typically found to require very intense laser fields, which could lead to the destruction of a condensed phase sample.

(4) The simulation and theoretical analysis of solution-phase coherent control is usually more complex and computationally intensive in comparison to gas-phase coherent control.

Nevertheless, an increasing number of recent studies that address various aspects of solution-phase coherent control is indicative of a growing interest in this fascinating problem. ${ }^{11-21}$ Some of the reasons behind this recent interest are the following:
(1) Many chemical and physical processes of fundamental as well as technological importance take place in solution.

(2) Finding the optimal pulse in complex systems has become possible in recent years via the experimental adaptive learning close loop strategy. ${ }^{8-10}$ Decoherence through coupling to intermolecular and intramolecular degrees of freedom is inevitable in these cases.

(3) It has been realized that optimal strategies can be devised that are based on lower intensities which will not destroy a condensed phase sample. ${ }^{22}$

(4) It is becoming increasingly clear that viewing decoherence as uncontrollable and destructive represents an oversimplification. Recent studies, mostly in the context of quantum computing, have demonstrated that decoherence can be controlled by laser fields, ${ }^{23,24}$ or by confining the system to decoherence free subspaces. ${ }^{25}$ Furthermore, it was demonstrated that decoherence is in fact indispensable for certain important objectives, such as cooling (the reduction of entropy) of internal molecular degrees of freedom. ${ }^{26-29}$

(5) Significant progress has been made during the last decade in our ability to simulate quantum molecular dynamics in the condensed phase. ${ }^{30,31}$

The purpose of this article is to quantitatively explore the interplay between coherent control and decoherence within the context of a simple model that allows for a tractable analytical treatment. The model is based on the laserinduced intramolecular asymmetrical hydrogen tunneling reaction, the control of which has been the subject of several recent papers. ${ }^{19-22,32}$ In these previous studies, the optimal pulse shape was calculated in the absence of decoherence, and its robustness to decoherence was explored. However, no attempt was made to optimize the pulse in the presence of decoherence. The performance of such an optimization is the main contribution of the present article, and provides a concrete demonstration of some of the general themes underlying the interplay between coherent control and decoherence. 


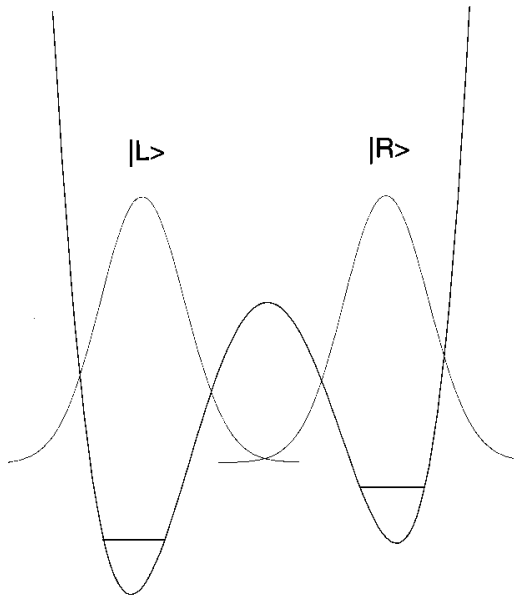

FIG. 1. A schematic view of the asymmetrical double well. Also shown are the localized stationary wave functions, $|R\rangle$ and $|L\rangle$.

The plan of this article is as follows. The basic model is outlined in Sec. II, and the optimal pulse in the absence of decoherence is presented. The optimal pulse in the presence of decoherence is discussed in Sec. III. This section also includes a general discussion on various possible dephasing mechanisms, and the optimization is carried out for two distinct types of pure dephasing. The main conclusions are summarized in Sec. IV.

\section{OPTIMIZATION OF HYDROGEN SWITCHING IN THE ABSENCE OF DECOHERENCE}

Following Refs. 19-22 and 32, we consider a generic intramolecular hydrogen transfer reaction that can be described in terms of a $1 \mathrm{D}$ reaction coordinate. The adiabatic potential energy along this reaction coordinate is assumed to take the form of an asymmetrical double-well, and the asymmetry is assumed large enough such that the ground and first excited wave functions are localized on both sides of the barrier. For concreteness, we will assume that the well to the left of the barrier is deeper than that to the right (cf. Fig. 1). The ground and first excited states are denoted $|L\rangle$ and $|R\rangle$, respectively $(|L\rangle$ is localized on the left-hand side and $|R\rangle$ on the right-hand side). It will be assumed that the energy gap between the $\{|R\rangle,|L\rangle\}$ doublet and higher lying states is much larger than the energy gap between $|L\rangle$ and $|R\rangle$, and that the temperature and laser pulse shape are such that contributions from these higher states are negligible. An example for a system that satisfies these conditions at room temperature when driven by a pulse of the shape described below is thioacetylacetone (in this case the hydrogen switches between an $\mathrm{OH}$ and a $\mathrm{SH}$ binding sites). ${ }^{32}$

The above discussion leads to a compact description of the reactive system in terms of the $2 \mathrm{D}$ Hilbert space spanned by $|R\rangle$ and $|L\rangle$. The field-free Hamiltonian can therefore be put in a form equivalent to that of a fictitious spin- $\frac{1}{2}$ system which is subject to a constant field in the $x z$ plane: ${ }^{33}$

$$
\mathbf{H}_{0}=-J \mathbf{S}_{\mathbf{x}}+A \mathbf{S}_{\mathbf{z}}
$$

where $A>0$ is the asymmetry, $J>0$ is the tunneling matrix element (both in units of angular frequency), ${ }^{34,35}$ and

$$
\begin{aligned}
& \mathbf{S}_{\mathbf{x}}=\frac{\hbar}{2}(|R\rangle\langle L|+| L\rangle\langle R|), \\
& \mathbf{S}_{\mathbf{y}}=\frac{\hbar}{2 i}(|R\rangle\langle L|-| L\rangle\langle R|), \\
& \mathbf{S}_{\mathbf{z}}=\frac{\hbar}{2}(|R\rangle\langle R|-| L\rangle\langle L|) .
\end{aligned}
$$

It should be noted that $A$ is assumed to be much larger than $J$, such that for all practical purposes, the localized states $|L\rangle$ and $|R\rangle$ are stationary (i.e., the mixing between $|L\rangle$ and $|R\rangle$ due to tunneling is negligible in the absence of a driving field).

The interaction of the reactive system with the driving laser field, $E(t)$, is given by

$$
\mathbf{W}(t)=-\boldsymbol{\mu} E(t),
$$

where $\boldsymbol{\mu}$ is the dipole operator, which is assumed to be linear in the reaction coordinate, q. We will assume, for simplicity, that $\langle R|\mathbf{q}| R\rangle=-\langle L|\mathbf{q}| L\rangle$ and that $\langle R|\mathbf{q}| L\rangle=0$. This assumption would be exact in the case of a symmetrical double-well. Deviations from it in the case of an asymmetrical doublewell will introduce an additional field-dependent term which is linear in $\mathbf{S}_{\mathbf{x}}$. Hence, the above assumption will be valid when this additional term is negligible relative to the fieldfree tunneling matrix element, $J .^{20}$ This assumption allows us to set $\boldsymbol{\mu}=\mu_{0} \mathbf{S}_{\mathbf{z}}\left(\mu_{0}\right.$ is a proportionality coefficient), and therefore rewrite $\mathbf{W}(t)$ in the following way:

$$
\mathbf{W}(t)=-\mathbf{S}_{\mathbf{z}} \epsilon(t),
$$

where $\epsilon(t)=\mu_{0} E(t)$ [ $\epsilon(t)$ has units of angular frequency].

We will assume that the system is prepared in the ground state, such that its initial state is given by the following density operator:

$$
\boldsymbol{\rho}(0)=|L\rangle\langle L|
$$

In terms of the spin- $\frac{1}{2}$ terminology, this means that $\left\langle\mathbf{S}_{\mathbf{z}}\right\rangle(0)$ $=-\hbar / 2$. Since, for all practical purposes, $|L\rangle$ is a stationary state, the system will remain in this state in the absence of a driving field. The laser is then turned on at $t=0$ in order to induce hydrogen transfer, i.e., to change the state to $|R\rangle$, which is localized on the other side of the barrier. Optimal control theory was utilized in Ref. 22 in order to find the pulse shape, $\epsilon(t)$, that maximizes the photo-isomerization yield after the pulse is turned off at $t=t_{p}, \operatorname{Tr}\left[\boldsymbol{\rho}\left(t_{p}\right)|R\rangle\right.$ $\times\langle R|]$, where $\boldsymbol{\rho}\left(t_{p}\right)$ is the density matrix at time $t=t_{p}$. In terms of the spin- $\frac{1}{2}$ terminology, this is equivalent to maximizing $\left\langle\mathbf{S}_{\mathbf{z}}\right\rangle\left(t_{p}\right)$. The actual optimal pulse shape obviously depends on the constraints imposed. Of particular importance to coherent control in the solution phase is the constraint imposed on the time-integrated intensity. This is because the pulse intensity has to be low enough so as not to destroy the sample. Such a constraint was indeed incorporated in Ref. 22 by penalizing high-intensity pulses in the objective functional. The optimal pulse at intensity levels which are not likely to destroy the sample was found to have the particularly simple form of a square pulse: 


$$
\epsilon(t)= \begin{cases}0 & \text { if } t<0 \\ \epsilon & \text { if } 0<t<t_{p} \\ 0 & \text { if } t>t_{p} .\end{cases}
$$

When the pulse is on, $0<t<t_{p}$, the total Hamiltonian is given by

$$
\mathbf{H}=\mathbf{H}_{\mathbf{0}}+\mathbf{W}(t)=-J \mathbf{S}_{\mathbf{x}}+(A-\boldsymbol{\epsilon}) \mathbf{S}_{\mathbf{z}} .
$$

Thus, the field tunes the asymmetry, and can therefore turn $|L\rangle$ into a nonstationary state. Starting with $\rho(0)$ $=|L\rangle\langle L|\left[\left\langle\mathbf{S}_{\mathbf{x}}\right\rangle(0)=\left\langle\mathbf{S}_{\mathbf{y}}\right\rangle(0)=0,\left\langle\mathbf{S}_{\mathbf{z}}\right\rangle(0)=-\hbar / 2\right], \quad$ it $\quad$ is straightforward to find $\left\langle\mathbf{S}_{\mathbf{z}}\right\rangle$ immediately after the pulse is turned off:

$$
\left\langle\mathbf{S}_{\mathbf{z}}\right\rangle\left(t_{p}, \boldsymbol{\epsilon}\right)=\frac{\hbar}{2}\left\{\frac{J^{2}}{E^{2}}\left[1-\cos \left(E t_{p}\right)\right]-1\right\},
$$

where

$$
E=\sqrt{(A-\epsilon)^{2}+J^{2}} .
$$

Maximizing the reaction yield, $\left\langle\mathbf{S}_{\mathbf{z}}\right\rangle\left(t_{p}, \boldsymbol{\epsilon}\right)$, with respect to the pulse length, $t_{p}$, for a given field amplitude, $\epsilon$, yields the following optimal pulse length and corresponding optimal yield:

$$
t_{p}^{*}(\boldsymbol{\epsilon})=\pi / E ; \quad\left\langle\mathbf{S}_{\mathbf{z}}\right\rangle\left[t_{p}^{*}(\boldsymbol{\epsilon}), \boldsymbol{\epsilon}\right]=\frac{\hbar}{2} \frac{J^{2}-(A-\boldsymbol{\epsilon})^{2}}{J^{2}+(A-\boldsymbol{\epsilon})^{2}} .
$$

Next, maximizing $\left\langle\mathbf{S}_{\mathbf{z}}\right\rangle\left[t_{p}^{*}(\boldsymbol{\epsilon}), \boldsymbol{\epsilon}\right]$ with respect to the pulse amplitude, $\epsilon$, yields the following optimal pulse amplitude and corresponding optimal yield:

$$
\epsilon^{*}=A, \quad\left\langle\mathbf{S}_{\mathbf{z}}\right\rangle\left[t_{p}^{*}\left(\epsilon^{*}\right), \epsilon^{*}\right]=\frac{\hbar}{2} .
$$

Thus, a square pulse with the optimized length and amplitude in Eqs. (10) and (11), respectively, which is consistent with the optimal pulse obtained via optimal control theory, leads to the maximal possible yield, i.e., to complete switching of the hydrogen from one side of the barrier to the other. Interestingly, $t_{p}^{*}\left(\epsilon^{*}\right)$ corresponds to the maximum of $t_{p}^{*}(\epsilon)$ as a function of $\epsilon$, i.e., the optimal pulse amplitude leads to the longest possible pulse. It should be noted that turning the field off at $t=t_{p}^{*}$ returns the system to its original Hamiltonian, $\mathbf{H}_{0}$, thereby locking it in the state $|R\rangle$.

The interpretation of how the optimal pulse operates is particularly straightforward in this case. To this end, it is instrumental to adopt a representation that diagonalizes the total Hamiltonian in Eq. (7):

$$
\begin{aligned}
& |-\rangle=\cos (\phi)|L\rangle+\sin (\phi)|R\rangle, \\
& |+\rangle=-\sin (\phi)|L\rangle+\cos (\phi)|R\rangle,
\end{aligned}
$$

where

$$
\tan (2 \phi)=J /(A-\epsilon) .
$$

The corresponding eigenvalues are $\pm \hbar E / 2$, such that $\mathbf{H}| \pm\rangle$ $= \pm(\hbar E / 2)| \pm\rangle$, with $E$ as in Eq. (9). In terms of the spin$\frac{1}{2}$ terminology, $\mathbf{H}$ corresponds to a spin $-\frac{1}{2}$ system which is subject to a constant magnetic field that lies in the $x z$ plane, and whose components along the $x$ and $z$ axes are $-J$ and $A-\epsilon$, respectively. The states $| \pm\rangle$ are the eigenstates of the spin component along the direction of the field, which can be identified as the $z$ axis of a new Cartesian coordinate system. The latter is obtained from the original coordinate system by rotating it by an angle $2 \phi$ around the $y$ axis. The spin components along the axes of the new coordinate system are related to these along the axes of the original coordinate system via

$$
\begin{aligned}
& \widetilde{\mathbf{S}}_{\mathbf{x}} \equiv \frac{1}{2}(|+\rangle\langle-|+|-\rangle\langle+|)=\cos (2 \phi) \mathbf{S}_{\mathbf{x}}+\sin (2 \phi) \mathbf{S}_{\mathbf{z}} \\
& \widetilde{\mathbf{S}}_{\mathbf{y}} \equiv \frac{1}{2 i}(|+\rangle\langle-|-|-\rangle\langle+|)=\mathbf{S}_{\mathbf{y}}, \\
& \widetilde{\mathbf{S}}_{\mathbf{z}} \equiv \frac{1}{2}(|+\rangle\langle+|-|-\rangle\langle-|)=-\sin (2 \phi) \mathbf{S}_{\mathbf{x}}+\cos (2 \phi) \mathbf{S}_{\mathbf{z}}
\end{aligned}
$$

In terms of the new coordinate system, the total Hamiltonian is given by

$$
\mathbf{H}=E \widetilde{\mathbf{S}}_{\mathbf{z}}
$$

The initial state can be described by a spinor, i.e., a 3D vector whose Cartesian components along the axes of the $\{\tilde{x} \tilde{y} \tilde{z}\}$ coordinate system are $\left\langle\widetilde{\mathbf{S}}_{\mathbf{x}}\right\rangle(0)=-\hbar J / 2 E,\left\langle\widetilde{\mathbf{S}}_{\mathbf{y}}\right\rangle(0)$ $=0$, and $\left\langle\widetilde{\mathbf{S}}_{\mathbf{z}}\right\rangle(0)=-\hbar(A-\boldsymbol{\epsilon}) / 2 E$, respectively. As is well known, the dynamics induced by $\mathbf{H}$ is equivalent to a normpreserving precession of the initial state vector around the $\tilde{z}$ axis, with the angular frequency $E$. Thus, the optimal pulse length, $t_{p}^{*}(\epsilon)$ [cf. Eq. (10)], corresponds to a half-cycle precession, which obviously leads to the maximal change in the spin component along the $z$ axis. Furthermore, the optimal pulse amplitude corresponds to setting the asymmetry to zero, cf. Eq. (11). Hence, the initial spinor is perpendicular to the axis of precession, which leads to complete switching of $\left\langle\mathbf{S}_{\mathbf{z}}\right\rangle$ from $-\hbar / 2$ to $\hbar / 2$.

The above simple scheme should be clearly distinguished from other methods of controlling tunneling in bistable systems, which are based on monochromatic or polychromatic CW driving fields. ${ }^{11-18}$ The main differences are as follows: (1) The method in the present article is based on a dc field, while the other methods are based on CW fields. (2) The method in the present article is based on manipulating the asymmetry, while methods such as coherent destruction of tunneling are based on tuning the effective tunneling matrix element. (3) The method in the present article is pulse based and the system remains in the product well after the pulse is turned off, while most other methods are based on a steady state which requires that the field stays on.

It should also be noted that although the overall effect of the optimal pulse is the same as that of a conventional $\pi$ pulse, the mechanism in the two cases is completely different. In the case of the optimal pulse, the driving field is used to shift the energy levels, whereas in the case of the conventional $\pi$ pulse, an oscillating field is employed which is in resonance with the transition. In fact, a conventional $\pi$ pulse cannot be realized in our model because it requires that the dipole moment be linear in $\mathbf{S}_{\mathbf{x}}$, rather than in $\mathbf{S}_{\mathbf{z}}$. 


\section{OPTIMIZATION OF HYDROGEN SWITCHING IN THE PRESENCE OF DECOHERENCE}

\section{A. General considerations}

The optimization scheme discussed in Sec. II assumes that the dynamics is unitary. In fact, since the initial state is pure, the analysis could have been carried out in terms of wave functions instead of density matrices. The discussion in Sec. II also clarifies the crucial role played by coherent dynamics in switching the hydrogen from one site to the other. In this sense, the system described provides one of the simplest examples for coherent control. However, the notion of an isolated reaction coordinate is a highly idealized one. In reality, the reaction coordinate will almost always be coupled to an external bath that consists of the other intramolecular and intermolecular degrees of freedom, and the coupling to this bath will have a dramatic effect on the system's dynamics. In particular, it is well known that coupling to a bath leads to dephasing, i.e., to the destruction of the very same coherences that make it possible to control the hydrogen tunneling in the first place. It is therefore natural to ask how the presence of dephasing affects the optimal pulse shape.

Generally speaking, the interplay between coherent control and dephasing can give rise to a rich variety of systemspecific phenomena. The hydrogen transfer system described above provides a relatively simple model for exploring such phenomena. This is because of the low dimensionality of the system, the simplicity of the optimal pulse in the absence of decoherence, and the fact that it does not explicitly involve time-dependent driving fields (except for switching the pulse on and off, which we assume to be instantaneous).

We will describe the decoherence dynamics in terms of a generic quantum master equation. We will also restrict the optimization to pulses that have the same general form as in Eq. (6). These choices obviously limit the range of validity of our results - it is not always possible to treat the dynamics in terms of a quantum master equation, and it is conceivable that the optimal pulse may have a different shape in the presence of dephasing. However, these choices also lead to the following major simplifications: (1) The fielddependence of the decoherent terms in the master equation is relatively straightforward in the case of a dc driving field, but becomes far more complicated in cases involving explicitly time-dependent driving fields. (2) Instead of solving the optimal control problem, the optimization is reduced to finding the optimal values of two parameters: the pulse length and amplitude (in the presence of dephasing). We believe that the model remains physically relevant even within this restricted setting.

Before embarking on the optimization of the pulse in the presence of decoherence, it is important to understand how the decoherence dynamics is modified by the laser field. We start by noting that the system-bath coupling term can be written in terms of field-free operators:

$$
\mathbf{H}_{\mathrm{bs}}=\boldsymbol{\Gamma}_{x} \otimes \mathbf{S}_{\mathbf{x}}+\boldsymbol{\Gamma}_{y} \otimes \mathbf{S}_{\mathbf{y}}+\boldsymbol{\Gamma}_{z} \otimes \mathbf{S}_{\mathbf{z}}
$$

where $\boldsymbol{\Gamma}_{x}, \boldsymbol{\Gamma}_{y}$, and $\boldsymbol{\Gamma}_{z}$ are bath operators, and $\mathbf{S}_{\mathbf{x}}, \mathbf{S}_{\mathbf{y}}$, and $\mathbf{S}_{\mathbf{z}}$ are as given in Eq. (2). It should be noted that $\boldsymbol{\Gamma}_{x}, \boldsymbol{\Gamma}_{y}$, and $\boldsymbol{\Gamma}_{z}$ may depend on the system parameters $A$ and $J$. We will assume that the system-bath coupling term in the presence of the driving field is the same as in its absence, such that $\boldsymbol{\Gamma}_{x}$, $\boldsymbol{\Gamma}_{y}$, and $\boldsymbol{\Gamma}_{z}$ in Eq. (16) are independent of the driving field.

Although the system-bath coupling in Eq. (16) is field independent, it can still be written in terms of fielddependent operators:

$$
\mathbf{H}_{\mathrm{bs}}=\widetilde{\boldsymbol{\Gamma}}_{x} \otimes \widetilde{\mathbf{S}}_{\mathbf{x}}+\widetilde{\boldsymbol{\Gamma}}_{y} \otimes \widetilde{\mathbf{S}}_{\mathbf{y}}+\widetilde{\boldsymbol{\Gamma}}_{\mathbf{z}} \otimes \widetilde{\mathbf{S}}_{\mathbf{z}}
$$

where $\widetilde{\mathbf{S}}_{\mathbf{x}}, \widetilde{\mathbf{S}}_{\mathbf{y}}$, and $\widetilde{\mathbf{S}}_{\mathbf{z}}$ are as given in Eqs. (14), and

$$
\begin{aligned}
& \widetilde{\boldsymbol{\Gamma}}_{x}=\cos (2 \phi) \boldsymbol{\Gamma}_{x}+\sin (2 \phi) \boldsymbol{\Gamma}_{z}, \\
& \widetilde{\boldsymbol{\Gamma}}_{y}=\boldsymbol{\Gamma}_{y}, \\
& \widetilde{\boldsymbol{\Gamma}}_{z}=-\sin (2 \phi) \boldsymbol{\Gamma}_{x}+\cos (2 \phi) \boldsymbol{\Gamma}_{z} .
\end{aligned}
$$

The decoherence dynamics during the period of time when the pulse is turned on will direct the system towards thermal equilibrium with respect to the field-dressed Hamiltonian, $\mathbf{H}=E \widetilde{\mathbf{S}}_{\mathbf{z}}$. Following the standard procedure (for example, see Ref. 36) leads to the following set of Heisenberg Markovian quantum master equations for $\widetilde{\mathbf{S}}_{\mathbf{x}}, \widetilde{\mathbf{S}}_{\mathbf{y}}$, and $\widetilde{\mathbf{S}}_{\mathbf{z}}$ :

$$
\begin{aligned}
& \dot{\tilde{\mathbf{S}}}_{\mathbf{x}}=-\frac{1}{T_{2}} \widetilde{\mathbf{S}}_{\mathbf{x}}-E \widetilde{\mathbf{S}}_{\mathbf{y}}, \\
& \dot{\tilde{\mathbf{S}}}_{\mathbf{y}}=E \widetilde{\mathbf{S}}_{\mathbf{x}}-\frac{1}{T_{2}} \widetilde{\mathbf{S}}_{\mathbf{y}}, \\
& \dot{\tilde{\mathbf{S}}}_{\mathbf{z}}=-\frac{1}{T_{1}}\left[\widetilde{\mathbf{S}}_{\mathbf{z}}-\left\langle\widetilde{\mathbf{S}}_{\mathbf{z}}\right\rangle_{\mathrm{eq}}\right],
\end{aligned}
$$

where

$$
\begin{aligned}
\frac{1}{T_{1}}= & \frac{1}{4}\left(1+e^{-\beta E}\right) \int_{-\infty}^{\infty} d \tau e^{i E \tau} \operatorname{Tr}\left\{\boldsymbol { \rho } _ { b } ^ { \mathrm { eq } } \left[\left(\widetilde{\boldsymbol{\Gamma}}_{x}(\tau)-i \widetilde{\boldsymbol{\Gamma}}_{y}(\tau)\right]\right.\right. \\
& \times\left[\left(\widetilde{\boldsymbol{\Gamma}}_{x}+i \widetilde{\boldsymbol{\Gamma}}_{y}\right]\right\},
\end{aligned}
$$

and

$$
\frac{1}{T_{2}}=\frac{1}{2 T_{1}}+\frac{1}{T_{2}^{*}}, \quad \frac{1}{T_{2}^{*}}=\frac{1}{2} \int_{-\infty}^{\infty} d \tau \operatorname{Tr}\left\{\boldsymbol{\rho}_{b}^{\mathrm{eq}} \widetilde{\boldsymbol{\Gamma}}_{z}(\tau) \widetilde{\boldsymbol{\Gamma}}_{z}\right\} .
$$

Here, $1 / T_{1}$ is the population relaxation rate constant, $1 / T_{2}$ is the dephasing rate constant, $1 / T_{2}^{*}$ is the pure dephasing rate constant, and $\boldsymbol{\rho}_{b}^{\mathrm{eq}}$ is the free bath equilibrium density matrix. It should be noted that the validity of Eqs. (19)-(21) rests on the following assumptions (see, for example, Ref. 36): (1) Weak coupling between the system and the bath; (2) separation of time scales between the rapidly decaying bath fluctuations and the relatively slow relaxation of the system; (3) the secular or rotating-wave approximation, which decouples the dynamics of $\mathbf{S}_{\mathbf{z}}$ from that of $\mathbf{S}_{\mathbf{x}}$ and $\mathbf{S}_{\mathbf{y}}$; and (4) the neglect of Lamb shifts.

The dependence of $T_{1}$ on the field comes from two sources: (1) the dependence of $\widetilde{\boldsymbol{\Gamma}}_{x}$ and $\widetilde{\boldsymbol{\Gamma}}_{y}$ on the field, through the angle $\phi\left[\right.$ cf. Eqs. (13) and (18)] and (2) $T_{1}$ is proportional to the Fourier component of the corresponding bath correlation function at the system frequency, $E$, which is field dependent [cf. Eqs. (9) and (20)]. As a result of the second effect, the dependence of $T_{1}$ on the field reflects the 
specifics of the system-bath interaction. On the other hand, the only field dependence of the pure dephasing rate constant, $1 / T_{2}^{*}$, is due to $\phi$ (the second source of fielddependence does not exist in this case because $1 / T_{2}^{*}$ is proportional to the zero frequency component of the corresponding correlation function). In order to avoid a model which is specific to the particular spectral density of the bath, we will assume that $1 / T_{2}^{*} \gg 1 / T_{1}$ and that $T_{1}$ processes are negligible on the time scale of the experiment $\left(t_{p}\right)$. It should also be noted that $T_{1}$ processes would rapidly thermalize the reactant and product populations, thereby making any control of the reaction quite impossible.

Focusing our attention on $T_{2}^{*}$, it should be reemphasized that $T_{2}^{*}$ will generally be field dependent. For example, the frequently encountered choice of a system-bath interaction term which is linear in the reaction coordinate $\left(\mathbf{S}_{\mathbf{z}}\right)$,

$$
\mathbf{H}_{\mathrm{bs}}=\boldsymbol{\Gamma}_{z} \otimes \mathbf{S}_{\mathbf{z}},
$$

would lead to $\widetilde{\boldsymbol{\Gamma}}_{z}=\cos (2 \phi) \boldsymbol{\Gamma}_{z}=\boldsymbol{\Gamma}_{z} \times(A-\epsilon) / \sqrt{(A-\epsilon)^{2}+J^{2}}$, such that

$$
\begin{aligned}
& \frac{1}{T_{2}^{*}} \rightarrow\left(\frac{1}{T_{2}^{*}}\right)_{a}=\gamma \times \frac{(A-\epsilon)^{2}}{(A-\epsilon)^{2}+J^{2}} ; \\
& \gamma=\frac{1}{2} \int_{-\infty}^{\infty} d \tau \operatorname{Tr}\left\{\boldsymbol{\rho}_{b}^{\mathrm{eq}} \boldsymbol{\Gamma}_{z}(\tau) \boldsymbol{\Gamma}_{z}\right\} .
\end{aligned}
$$

While $\gamma$ in Eq. (23) is field independent, the factor preceding it is explicitly field dependent. It is important to note that in this case, $1 / T_{2}^{*}=0$ when $\epsilon=A$. This is because the coupling to the bath, $\boldsymbol{\Gamma}_{z} \otimes \mathbf{S}_{z}$, is off-diagonal within the eigenrepresentation of the Hamiltonian, $\mathbf{H}=-J \mathbf{S}_{\mathbf{x}}$, and is therefore unable to lead to pure dephasing (it can still lead to population relaxation, which is assumed negligible). Thus, it will be advantageous to minimize $|A-\epsilon|$ not only because it is the best strategy in the absence of decoherence [cf. Eq. (11)], but also because it diminishes the dephasing rate. In other words, the optimal laser field can be actively used to eliminate dephasing, as well as steering the coherent dynamics towards the desired goal!

However, there will also be cases where $T_{2}^{*}$ is field independent. This happens when the interaction term is as in Eq. (16), where $\boldsymbol{\Gamma}_{x}, \boldsymbol{\Gamma}_{y}$, and $\boldsymbol{\Gamma}_{z}$ are assumed to be uncorrelated and to have identical autocorrelation functions:

$$
\operatorname{Tr}\left\{\boldsymbol{\rho}_{b}^{\mathrm{eq}} \boldsymbol{\Gamma}_{i}(t) \boldsymbol{\Gamma}_{j}\right\}=\delta_{i, j} C(t), \quad i, j=x, y, z .
$$

In this case,

$$
\frac{1}{T_{2}^{*}} \rightarrow\left(\frac{1}{T_{2}^{*}}\right)_{b}=\frac{1}{2} \int_{-\infty}^{\infty} d \tau C(\tau)
$$

is field independent and therefore cannot be controlled by pulse shaping.

Equations (23) and (25) represent two out of a very wide range of possibilities that will emerge when coherent control is attempted in the solution phase. The above discussion makes it clear that the optimal pulse design would simultaneously optimize the decoherent and coherent components of the dynamics. In fact, the optimal pulse will often represent a compromise between these two driving forces. It should also

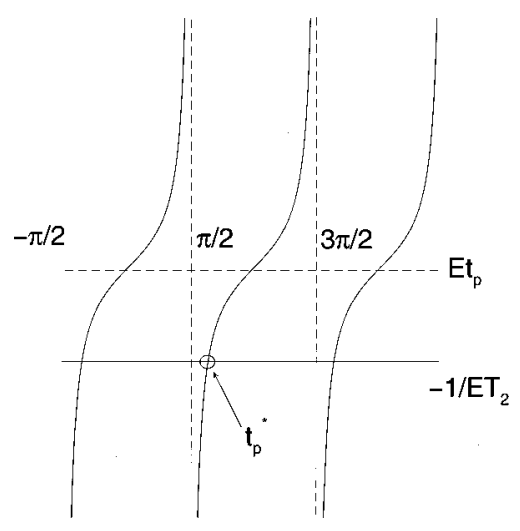

FIG. 2. Optimization of the pulse length. The optimal length, $t_{p}^{*}(\epsilon)$, corresponds to the smallest positive value of $t_{p}$ where the functions $\tan \left(E t_{p}\right)$ and $1 / E T_{2}$ cross (circled).

be noted that the optimal pulse will depend on the specific decoherence mechanism that is at play, which provides another mean of control in the sense of looking for a system with an optimal decoherence mechanism. In the next two sections we will demonstrate these points in the context of the two decoherence mechanisms described above.

\section{B. Optimization with respect to the pulse width}

Neglecting $T_{1}$ relaxation $\left(T_{1} \rightarrow \infty\right)$ and solving Eqs. (19) for the initial state in Eq. (5) leads to the following expression for the final yield:

$$
\left\langle\mathbf{S}_{\mathbf{z}}\right\rangle\left(t_{p}, \boldsymbol{\epsilon}\right)=\frac{\hbar}{2}\left\{\frac{J^{2}}{E^{2}}\left[1-\cos \left(E t_{p}\right) e^{-t_{p} / T_{2}}\right]-1\right\} .
$$

It is straightforward to maximize the yield, $\left\langle\mathbf{S}_{\mathbf{z}}\right\rangle\left(t_{p}, \boldsymbol{\epsilon}\right)$, with respect to the pulse length, $t_{p}$. The optimal pulse length, $t_{p}^{*}$, is found to satisfy the following identity:

$$
\tan \left(E t_{p}^{*}\right)=-\frac{1}{E T_{2}}
$$

Equation (27) has an infinite number of solutions. However, since $E, T_{2}$ and $t_{p}^{*}$ are all positive, the optimal pulse length corresponds to the smallest positive solution for $t_{p}^{*}$ (cf. Fig. 2), namely,

$$
t_{p}^{*}(\epsilon)=\frac{1}{E}\left[\pi-\tan ^{-1}\left(\frac{1}{E T_{2}}\right)\right] .
$$

The dependence of $t_{p}^{*}(\epsilon)$ on the dephasing rate is shown in Fig. 3. As $E T_{2} \rightarrow \infty, t_{p}^{*}(\epsilon)$ coincides with its value in the absence of dephasing, namely $t_{p}^{*}(\epsilon) \rightarrow \pi / E$, which corresponds to the maximal possible yield [cf. Eq. (10)]. A finite value of $E T_{2}$ leads to a reduction in $t_{p}^{*}(\epsilon)$. This reduction represents a compromise between the opposing tendencies of coherent dynamics, represented by the precession frequency $E$, and dephasing, represented by $1 / T_{2}$. This is because a longer pulse width, as favored by coherent dynamics, also leads to more dephasing. It should be noted that the system still contains some coherence between the reactant and product states at time $t_{p}\left(\left\langle\mathbf{S}_{\mathbf{x}}\right\rangle\left(t_{p}\right),\left\langle\mathbf{S}_{\mathbf{y}}\right\rangle\left(t_{p}\right) \neq 0\right)$. However, 


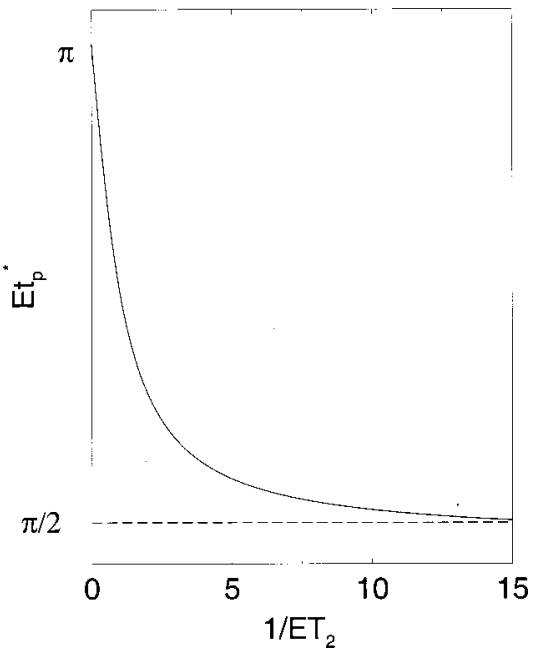

FIG. 3. The optimal pulse length, $t_{p}^{*}(\epsilon)$, as a function of the dephasing rate, $1 / T_{2}$.

switching off the field will stabilize $\left\langle\mathbf{S}_{\mathbf{z}}\right\rangle$ at its value at $t$ $=t_{p}$, while $\left\langle\mathbf{S}_{\mathbf{x}}\right\rangle$ and $\left\langle\mathbf{S}_{\mathbf{y}}\right\rangle$ will gradually vanish via pure dephasing.

When $E T_{2}$ becomes very small, the optimal pulse length approaches the lower bound, $t_{p}^{*}(\epsilon)=\pi / 2 E$, i.e., the optimal pulse becomes a quarter-cycle rather than a half-cycle pulse. However, it should be noted that $\left\langle\mathbf{S}_{\mathbf{z}}\right\rangle\left(t_{p}, \boldsymbol{\epsilon}\right)$ is actually independent of $t_{p}$ in this case, and that the optimal yield, $\left\langle\mathbf{S}_{\mathbf{z}}\right\rangle\left(t_{p}^{*}(\boldsymbol{\epsilon}), \boldsymbol{\epsilon}\right)=-(A-\boldsymbol{\epsilon})^{2} / 2 E^{2}$, is completely dictated by the dephasing. Nevertheless, this yield still depends on the field amplitude, $\epsilon$, and can be optimized with respect to it. Figure 4 provides a schematic view of the above observations for the case $A=\epsilon$.

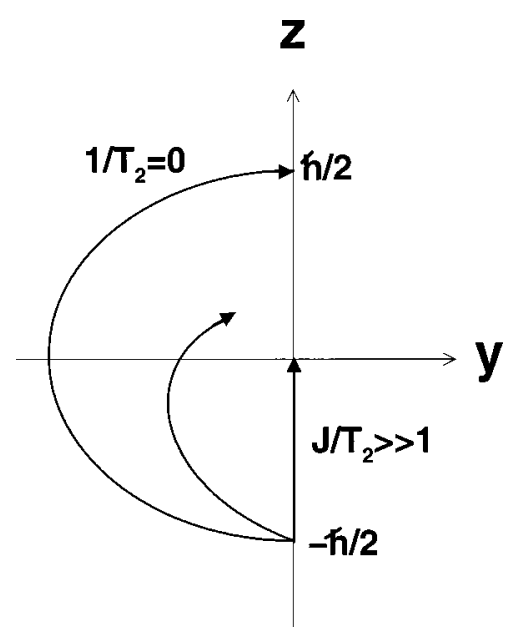

FIG. 4. A schematic view of the control scheme for $A=\epsilon$. In this case the precession of the spinor takes place in the $y z$ plane, around the $x$ axis. In the absence of dephasing, $1 / T_{2}=0$, the optimal pulse leads to a precession by half a cycle. In the opposite extreme of strong dephasing, $J / T_{2} \gg 1$, the precession is completely damped, and the increase in $\left\langle\mathbf{S}_{\mathbf{z}}\right\rangle$ is brought about solely by the dephasing process. As a result, $\left\langle\mathbf{S}_{\mathbf{z}}\right\rangle=0$ is the maximum obtainable yield. In the intermediate case, the optimal pulse is shorter than it would have been in the absence of dephasing, and therefore leads to a precession between half a cycle and a quarter of a cycle. The corresponding yield is between 0 and $\frac{1}{2}$.

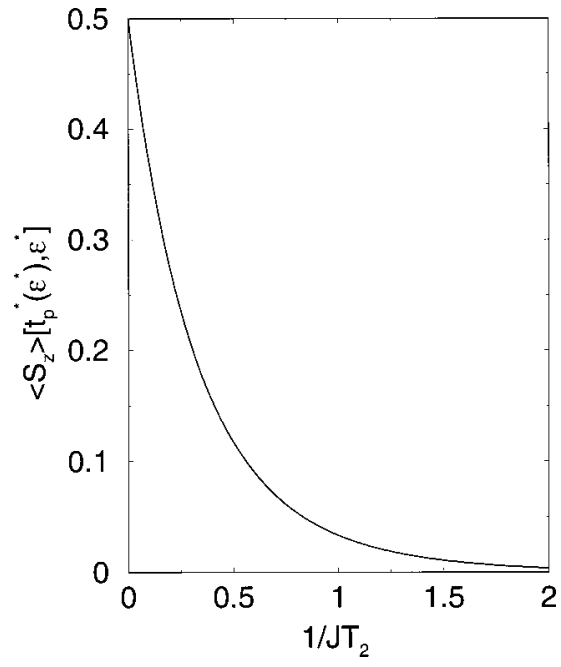

FIG. 5. The optimal yield, $\left\langle\mathbf{S}_{\mathbf{z}}\right\rangle\left[t_{p}^{*}\left(\epsilon^{*}\right), \epsilon^{*}\right]$, as a function of the dephasing rate, $1 / T_{2}$.

\section{Optimization with respect to the pulse amplitude}

Our next step will be to evaluate the optimal field amplitude, $\epsilon^{*}$. Substituting Eq. (28) into Eq. (26), we find that $\left\langle\mathbf{S}_{\mathbf{z}}\right\rangle\left[t_{p}^{*}(\boldsymbol{\epsilon}), \boldsymbol{\epsilon}\right]$ can be put in the following form:

$$
\left\langle\mathbf{S}_{\mathbf{z}}\right\rangle\left[t_{p}^{*}(\boldsymbol{\epsilon}), \boldsymbol{\epsilon}\right]=\frac{1}{2}\left[J^{2} T_{2}^{2} F\left(1 / E T_{2}\right)-1\right],
$$

where

$$
F(x)=x^{2}\left\{1-\cos \left[\pi-\tan ^{-1}(x)\right] e^{-x\left(\pi-\tan ^{-1}(x)\right)}\right\} .
$$

In the case where $T_{2}$ is independent of $\epsilon$, Eq. (25), the dependence of $\left\langle\mathbf{S}_{\mathbf{z}}\right\rangle\left[t_{p}^{*}(\boldsymbol{\epsilon}), \boldsymbol{\epsilon}\right]$ on $\boldsymbol{\epsilon}$ is contained in the variable $1 / E T_{2}$. Since $F(x)$ is a monotonically increasing function of $x$, maximizing $\left\langle\mathbf{S}_{\mathbf{z}}\right\rangle\left[t_{p}^{*}(\boldsymbol{\epsilon}), \boldsymbol{\epsilon}\right]$ with respect to $\epsilon$ is equivalent to maximizing $1 / E T_{2}$ with respect to $\epsilon$, which implies that

$$
\epsilon^{*}=A \text {. }
$$

Thus, the optimal pulse amplitude is the same as that in the absence of dephasing. Substituting this $\epsilon^{*}$ back into Eq. (29) leads to the following optimal yield:

$$
\begin{aligned}
\left\langle\mathbf{S}_{\mathbf{z}}\right\rangle\left[t_{p}^{*}\left(\epsilon^{*}\right), \epsilon^{*}\right]= & -\frac{1}{2} \cos \left[\pi-\tan ^{-1}\left(\frac{1}{|J| T_{2}}\right)\right] \\
& \times e^{-\left(1 /|J| T_{2}\right)\left[\pi-\tan ^{-1}\left(1 /|J| T_{2}\right)\right]} .
\end{aligned}
$$

In Fig. 5, the optimal yield, $\left\langle\mathbf{S}_{\mathbf{z}}\right\rangle\left[t_{p}^{*}\left(\epsilon^{*}\right), \epsilon^{*}\right]$, is plotted as a function of the dephasing rate, $1 / T_{2}$. The optimal yield is clearly lower than $\frac{1}{2}$ in the presence of dephasing, and has a lower bound of 0 , which is the optimal yield in the presence of very strong dephasing, i.e., when $1 /|J| T_{2} \gg 1$.

It should be noted that when $\epsilon=\epsilon^{*}=A$, the dephasing and coherent components of the dynamics push the system in the same direction. We have already seen that the coherent component of the dynamics favors this amplitude because the initial spinor is then perpendicular to the axis of precession, thereby allowing for complete switching. At the same time, the dephasing process destroys the components of the initial spinor that are perpendicular to the axis of precession. Thus, setting the axis of precession along the $x$ axis means that the dephasing increases $\left\langle\mathbf{S}_{\mathbf{z}}\right\rangle$ during the first quarter 
cycle, by making it less negative. Because the second stage of the pulse involves a precession by less than a quarter of a cycle, the net effect is that dephasing actually helps to increase the yield!

We now turn to the case where $T_{2}$ is explicitly dependent on $\epsilon$, Eq. (23). It is straightforward to verify that here, too, $\left\langle\mathbf{S}_{\mathbf{z}}\right\rangle\left(t_{p}^{*}(\boldsymbol{\epsilon}), \boldsymbol{\epsilon}\right)$ is maximized when $\boldsymbol{\epsilon}^{*}=A$. However, unlike the case of a field-independent $T_{2}$, in this case the optimal yield is given by

$$
\left\langle\mathbf{S}_{\mathbf{z}}\right\rangle\left(t_{p}^{*}(\boldsymbol{\epsilon}), \epsilon^{*}\right)=\frac{1}{2},
$$

which is the same as that in the absence of dephasing. In other words, we can reach complete switching in a system which is subject to dephasing. The origin of this result can be traced back to the fact that by setting $\epsilon=A$, we have also effectively neutralized the dephasing mechanism $\left[\left(1 / T_{2}\right)(\epsilon\right.$ $=A)=0$, cf. Eq. (23)].

\section{CONCLUSIONS}

The goal of this article was to provide a concrete and simple demonstration of several unique features of coherent control in the presence of decoherence. The main observations may be summarized as follows:

(1) Achieving coherent control in the solution phase is as much about controlling dephasing as it is about steering the coherent dynamics. The optimal pulse shape will generally represent the best compromise between these two driving forces.

(2) The optimal pulse shape in the presence of decoherence will usually be different from the optimal pulse shape in the absence of decoherence.

(3) The optimal pulse and optimal yield can be extremely sensitive not only to the system's free Hamiltonian, but also to the nature of the dephasing mechanism. Both dephasing mechanisms discussed above can be realized in solution. Mechanism $a$ will be relevant when the bath couples predominantly to the reaction coordinate, and as a result leads to fluctuations of the asymmetry. Mechanism $b$ will be relevant when the bath also causes similar fluctuations in the tunneling matrix element (by modifying the barrier height and well frequencies). In fact, one should expect other intermediate cases between these two extremes. Furthermore, it appears that coherent control experiments can be used to extract quantitative information about the particular dephasing mechanisms that are at play in a given system.

It should be noted that the features demonstrated in this article are expected to be general and not limited to the par- ticular model studied. The study of more elaborate models of coherent control in the presence of decoherence will be the subject of future work.

\section{ACKNOWLEDGMENTS}

The author would like to thank Professor Ronnie Kosloff and Professor David Tannor for helpful suggestions. This project was supported by the National Science Foundation FOCUS Center, Grant No. 0114336, and by startup funding from the University of Michigan.

${ }^{1}$ D. J. Tannor and S. A. Rice, Adv. Chem. Phys. 70, 441 (1988).

${ }^{2}$ R. J. Gordon and S. A. Rice, Annu. Rev. Phys. Chem. 48, 601 (1997).

${ }^{3}$ H. Rabitz and W. Zhu, Acc. Chem. Res. 33, 572 (2000).

${ }^{4}$ S. A. Rice and M. Zhao, Optical Control of Molecular Dynamics (Wiley, New York, 2000).

${ }^{5}$ R. Kosloff et al., Chem. Phys. 139, 201 (1989).

${ }^{6}$ W. Zhu, J. Botina, and H. Rabitz, J. Chem. Phys. 108, 1953 (1998).

${ }^{7}$ Y. Ohtsuki, W. Zhu, and H. Rabitz, J. Chem. Phys. 110, 9825 (1999).

${ }^{8}$ A. Assion et al., Science 282, 919 (1998).

${ }^{9}$ R. J. Levis, G. M. Menkir, and H. Rabitz, Science 292, 709 (2001).

${ }^{10}$ B. J. Pearson, J. L. White, T. C. Weinacht, and P. H. Bucksbaum, Phys. Rev. A 63, 063412 (2001).

${ }^{11}$ F. Grossmann, T. Dittrich, P. Jung, and P. Hänggi, Phys. Rev. Lett. 67, 516 (1991)

${ }^{12}$ R. Bavli and H. Metiu, Phys. Rev. Lett. 69, 1986 (1992).

${ }^{13}$ D. G. Evans, R. D. Coalson, H. J. Kim, and Y. Dakhnovskii, Phys. Rev. Lett. 75, 3649 (1995).

${ }^{14}$ D. E. Makarov and N. Makri, Phys. Rev. B 52, R2257 (1995).

${ }^{15}$ N. Makri, J. Chem. Phys. 106, 2286 (1997).

${ }^{16}$ N. Makri and L. Wei, Phys. Rev. E 55, 2475 (1997).

${ }^{17}$ R. I. Cukier, C. Denk, and M. Morillo, Chem. Phys. 217, 179 (1997).

${ }^{18}$ M. Grifoni and P. Hänggi, Phys. Rep. 304, 229 (1998).

${ }^{19}$ O. Kühn, Eur. Phys. J. D 6, 49 (1999).

${ }^{20}$ H. Naundorf, K. Sundermann, and O. Kühn, Chem. Phys. 240, 163 (1999).

${ }^{21}$ O. Kuhn, Y. Zhao, F. Shuang, and Y. Yan, J. Chem. Phys. 112, 6104 (2000).

${ }^{22}$ N. Dŏslić, O. Kühn, J. Manz, and K. Sundermann, J. Phys. Chem. A 102, 9645 (1998).

${ }^{23}$ D. Vitali and P. Tombesi, Phys. Rev. A 59, 4178 (1999).

${ }^{24}$ C. Search and P. R. Berman, Phys. Rev. Lett. 85, 2272 (2000).

${ }^{25}$ D. Bacon, J. Kempe, D. A. Lidar, and K. B. Whaley, Phys. Rev. Lett. 85, 1758 (2000)

${ }^{26}$ A. Bartana, R. Kosloff, and D. J. Tannor, J. Chem. Phys. 99, 196 (1993).

${ }^{27}$ A. Bartana, R. Kosloff, and D. J. Tannor, J. Chem. Phys. 106, 1435 (1997).

${ }^{28}$ D. J. Tannor and A. Bartana, J. Phys. Chem. A 103, 10359 (1999).

${ }^{29}$ D. J. Tannor, R. Kosloff, and A. Bartana, Faraday Discuss. 113, 365 (1999)

${ }^{30}$ N. Makri, Annu. Rev. Phys. Chem. 50, 167 (1999).

${ }^{31}$ P. Jungwirth and R. B. Gerber, Chem. Rev. 99, 1583 (1999)

${ }^{32}$ N. Dǒslić et al., Phys. Chem. Chem. Phys. 1, 1249 (1999).

${ }^{33}$ C. Cohen-Tannoudji, B. Diu, and F. Laloë, Quantum Mechanics (Wiley, New York, 1977).

${ }^{34}$ W. A. Phillips, in Amorphous Solids and the Liquid State, edited by N. March, R. Street, and A. Tosi (Plenum, New York, 1985), p. 467.

${ }^{35}$ J. L. Skinner and H. P. Trommsdorf, J. Chem. Phys. 89, 897 (1988).

${ }^{36}$ E. Geva, R. Kosloff, and J. L. Skinner, J. Chem. Phys. 102, 8541 (1995). 